Schreiben des Herrn Professors Plantamour, Directors der Sternwarte in Genf, an den Herausgeber. Genève 1844. Férrier 9.

Monsieur,

Jai encore à vous communiquer deux nouvelles positions de la comète, que j'ai observées depuis ma dernière lettre, savoir.

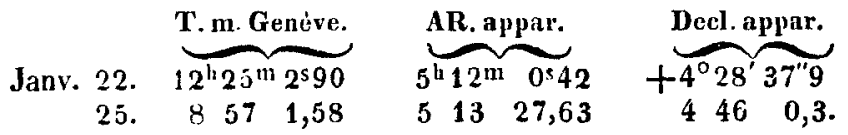

La faiblesse de la comete pour ces dernières observations et la rapidité de son éloignement de la terre me font craindre de ne plus pouvoir obtenir de nouvelles positions.

J'ai calculé les ćléments elliptiques de l'orbite avec l'ob. servation de Paris du 24 Novembre, et celles que j'a faites le 17 Décembre et le 18 Janvier; voici ce que j'ai trouvé:

Long. moy.époque 1 єr Janv.1844, à Midi

t. m. Paris............ 59 $39^{\circ} 47^{\prime \prime 67}$ (l'équ.moy.

Longitude Périhélie............49 29 38,30 1 Janv.

Longitude Noud .............20931 14,5 1844.

Inclinaison............... $1122 \quad 17,3$

Angle dont le Sinus $=$ Excentricité... $3346 \quad 37,8$

Demi grand axc............ 3,80801

Moyen mouvement diurne.......477448367

Durée de la revolution........... 7ans4310

Mouvement direct.
Ces éléments représentent de la manière suivante les obser. vations, en tenant compte de l'aberration et de la parallaxe de la comète; les signes affectés aux erreurs en longitude et en latitude indiquent l'excis des positions observées sur les positions calculées :

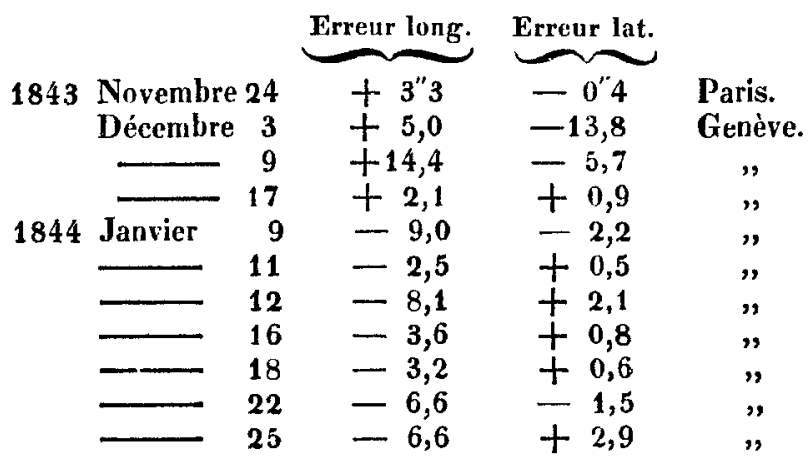

Ces éléments représentent assez bien les observations; peutêtre pourrait-on liminuer encore les erreurs en faisant concourir toutes les positions observées à la correction des éléments par la méthode des moindres carrés; c'est ce que j'es. sayerai peut-être, après avoir terminé quelques travaux plus pressés.

\title{
E. Plantamour:
}

\section{Schreiben des Herrn Hind an den Herausgeber. 1844 February 9.}

The following elliptical elements of M. Faye's Comet, I have computed from the Paris observation on Nov. 24, Mr. Rümker's on Dec. 17 and one by Sir James South at Kensington on Jan. 15. These elements represent the middle observation with errors of a few seconds in longitude and latitude.

Epoche 1844 January 1,0 Mean Time at Greenwich.

$\begin{array}{rrrr}\text { Mean Anomaly ........... } & 9^{\circ} 56^{\prime} & 366^{\prime \prime} 67 \\ \text { Longitude of the Perihelion..... } 53 & 19 & 52,35 \\ \text { Longitude of the Ascending Node } & 208 & 24 & 18,26 \\ \text { Inclination...... } & 11 & 7 & 8,70 \\ \text { Angle of excentricity }\left(=\sin { }^{\prime} \text { e) }\right. & 31 & 54 & 52,15\end{array}$

Log. semi - axis major........ 0,5582124

Log. semi-axis minor... . . . . . 0,4870372

Log. semi - parameter.......... 0,4158620

Lug. perihelion distance....... 0,2315531

Mean daily sidereal motion.... 516"04548

Period of Sidereal Revolution ... $2511^{\text {days } 403}$ or 6 years Motion direct. 11 months.

Log. $V a(1+e)=0,3712607$ Log. $V a(1-e)=0,1157766$ Log. Excentricity in seconds $=5,0375958$.

I calculated these elements agreeably to the general mothod by Professor Gauss.

\section{John Russell Hind.}

Elements of several aucient Comets.

These orbits are founded on the chinese observations publisbed in the appendix to the "Connaissance des Temps" for 1846 by M. Edouard Biot. With such data as are furnished by these observations the elements cannot be expected to be more than rough approximations.
The orbit of the second comet of 568 is calculated upon the later observations. About the end of September the comet was at a distance from the earth, equal to 0,13 of the mean distance of the earth from the sun, according to my elements. 
The elements of the comet of 574 are very uncertain. Those of the great comet in 1337 are more satisfactory: they represent the comet's apparent path as accurately as I could hope from such imperfect observations.

The orbit of the comet of 1385 is, I think, more certain than either that of 568 or 574 .

\begin{tabular}{|c|c|c|c|c|}
\hline & 568 & 574 & & 1385 \\
\hline $\begin{array}{c}\text { erih. Pass.Greenw } \\
\text { M. T. Old Style }\end{array}$ & & & & \\
\hline Long. of Perihelion & $316^{\circ} 47^{\prime}$ & $143^{\circ} 39^{\prime}$ & $350^{\circ} 22^{\prime}$ & $101^{\circ} 47$ \\
\hline f Asc. Node & 29436 & 12817 & 996 & 26831 \\
\hline Incliuation & & 4631 & 4254 & $\begin{array}{ll}52 & 15\end{array}$ \\
\hline og. Per. D & 9,9491 & 9,9 & 162 & \\
\hline & & & & \\
\hline
\end{tabular}

John Russell Hind.

Dr. Goldschmidts dritte elliptische Elemente des von Herrn Faye entdeckten Cometen.

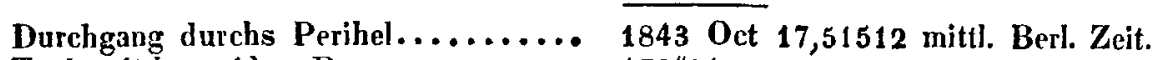

Tägl. mittlere sider. Bewegung ...... 479" 8425

Log. der halben grofsen Axe....... 0,5792721

Excentrieitü................. 0,5541125

Excentricitätswinkel............ $33^{\circ} 38^{\prime} 58^{\prime \prime} 4$

Perihel................... 494457,9 bezogen aufs mittl. Aequin. 1844 Jan. 0

Knoten................... 209267,8$\}$ und siderisch ruhend vorausgesetzt.

Neigung der Bahn............ 112128,4

Siderische Umlaufszeit........... 2700.88 Tage.

Vergleichung der Elemente mit den Beobachtungen. Rectascension Declination.

1843 Novbr. 24

$$
26
$$

\section{7}

28

29

Decbr. 1

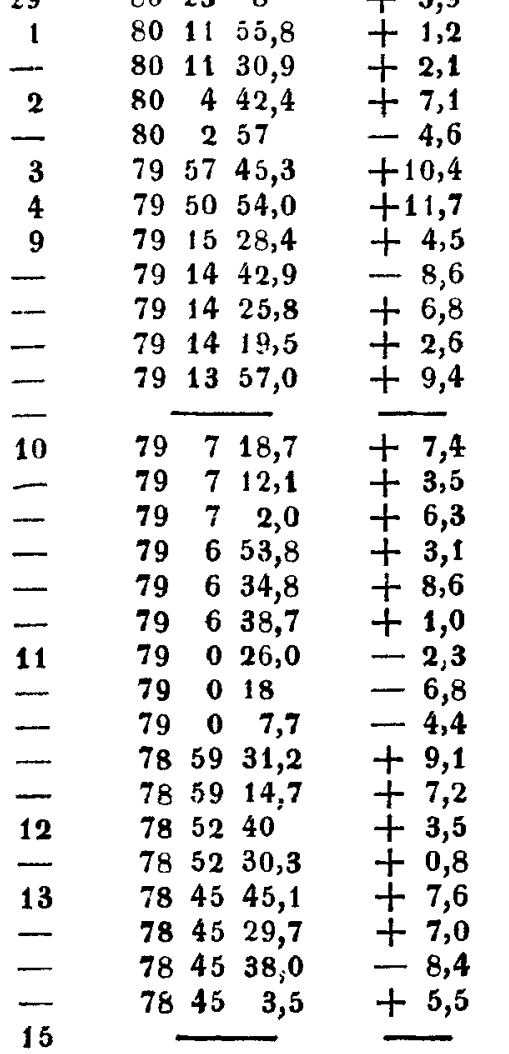

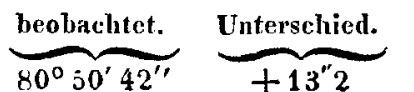

$\begin{array}{llll}80 & 42 & 22\end{array}$

$\begin{array}{llll}80 & 36 & 33\end{array}$

$80 \quad 30 \quad 45$

$\begin{array}{lll}80 & 23 & 8\end{array}$

01155

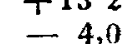

$\begin{array}{r}1,3 \\ \hline\end{array}$

$-0,2$

3,9
$+\quad 19$

$+1,2$
$+\quad 2$,

$+2,1$

$+7,6$
$-\quad 4,6$

$+10,4$

$+11,7$
$+\quad 4,5$

$-8,6$

$+6,8$

$+2,6$

$+9,4$

$\begin{array}{r}7,4 \\ +\quad 3,5 \\ \hline\end{array}$

$+3$

$+8,6$

$+1,0$
$-2,3$

$-6,8$

$-4,4$

$+7,2$

7,6

8,4

$+5,5$

\begin{tabular}{|c|c|c|c|c|}
\hline beob & acht & & Interschied. & Beobaelute \\
\hline$-6^{0}$ & 30 & 35 & $-11,8$ & Paris \\
\hline 6 & 9 & 44 & $+1,3$ & Paris \\
\hline 5 & 57 & 42 & $+1,4$ & Paris \\
\hline 5 & 46 & 41 & $-15,1$ & Paris \\
\hline 5 & 33 & 12 & $-1,1$ & Paris \\
\hline 5 & 14 & 44,4 & $-3,7$ & Hamburg \\
\hline 5 & 13 & 49,8 & $+6,7$ & Altona \\
\hline 5 & 3 & 57,2 & $+0,5$ & Bonn \\
\hline 5 & 1 & 40 & $-2,0$ & Paris \\
\hline 4 & 54 & 28,5 & $+7,3$ & Genf \\
\hline 4 & 45 & 25,6 & $+6,7$ & Altona \\
\hline 4 & $\mathbf{5}$ & 49,1 & $-5,8$ & Genf \\
\hline 4 & 4 & 39,1 & $+11,2$ & Hamburg \\
\hline 4 & 4 & 33,3 & $+11,2$ & Altona \\
\hline 4 & 4 & 40,0 & $-0,5$ & Berlin \\
\hline 4 & 4 & 19,3 & $+6,0$ & Altona (Merid. -Beob.) \\
\hline 4 & 4 & 30,6 & $-5,4$ & Hamburg (Merid. - Beob.) \\
\hline 3 & 58 & 8,2 & $-3,4$ & Hamburg \\
\hline $\mathbf{3}$ & 58 & 1,3 & $-5,6$ & Bonn \\
\hline $\mathbf{3}$ & 57 & 52,6 & $-2,7$ & Altona \\
\hline $\mathbf{3}$ & $5 ?$ & 39,5 & $+0,6$ & Berlin \\
\hline 3 & 57 & 35,6 & $-7,1$ & Altona (Merid. - Beob.) \\
\hline $\mathbf{3}$ & 57 & 30,7 & $-5,1$ & Bonn (Merid. - Beob.) \\
\hline 3 & 51 & 46,9 & $-1,7$ & Bonn \\
\hline $\mathbf{3}$ & 51 & 46 & $-11,8$ & Paris \\
\hline 3 & 51 & 37,2 & $-8,3$ & Hamburg \\
\hline 3 & 51 & 16,1 & $-5,9$ & Altona \\
\hline 3 & 50 & 59,2 & $-3,6$ & Altona (Merid. . Beob.) \\
\hline 3 & 45 & 35 & $-18,7$ & Paris \\
\hline 3 & 45 & 12,5 & $-4,7$ & Bonn \\
\hline 3 & 39 & 56,7 & $-8,7$ & Hamburg \\
\hline 3 & 39 & 44,7 & $-6,4$ & Altona \\
\hline 3 & 39 & 28,6 & $+4,4$ & Göttingen \\
\hline $\mathbf{3}$ & 39 & 12,3 & $+4,3$ & Berlin \\
\hline $\mathbf{3}$ & 28 & 40,8 & +42 & Hamburg (Merid.-Beob.) \\
\hline
\end{tabular}

